\title{
Vertuschung, Verantwortung, Wiederverwendung? Weshalb sich einfache Antworten verbieten
}

\author{
Ein Beispiel aus dem Erzbistum Freiburg (1924-1936)
}

\author{
Dominik Burkard
}

Der hier skizzierte Fall stammt aus dem Erzbistum Freiburg und liegt mit seiner Verortung in den 1920er und 1930er Jahren am weitesten zurück. ${ }^{1}$ Er wurde nicht erhoben im Rahmen eines aktuellen Aufarbeitungsprojektes, sondern begegnete dem Verfasser bei anders gelagerten Studien zur nationalsozialistischen Kirchenpolitik und zur jüngst wieder vieldiskutierten Rolle des Freiburger Erzbischofs Conrad Gröber (1872-1948). ${ }^{2}$ Es handelt sich um einen von insgesamt etwa ein Dutzend Fällen, die der badischen Gestapo bei ihren weitgespannten Recherchen zu ,Sittlichkeitsdelikten' von Klerikern in die Hände fielen. ${ }^{3}$ Damit schließt der Beitrag zeitlich und kontextuell unmittelbar an die von Dagmar Lieske und Hans Günter Hockerts herausgearbeiteten Zusammenhänge an. ${ }^{4}$ Konkret geht es um das etwa ein Jahr dauernde Verhältnis eines 30-jährigen Priesters zu einem 20-jährigen, nach damaligem Verständnis noch minderjährigen und unter Aufsicht der Fürsorge stehenden, also sozial und psychisch nicht gefestigten Mädchen. Die ersten Sexualkontakte müssen wohl als Vergewaltigung betrachtet werden. ${ }^{5}$

1 Diese Untersuchung stützt sich auf Quellen aus dem Erzbischöflichen Archiv Freiburg (EAF), dem Staatsarchiv Freiburg (StAF) und dem Generallandesarchiv Karlsruhe (GLA).

2 Vgl. Dominik Burkard, Das "Dossier Gröber" in den Archives de l'occupation. Zu Wert und Wahrheitsgehalt eines problematischen Quellenbestands, in: Hans-Otto Mühleisen/ Dominik Burkard, Erzbischof Conrad Gröber reloaded. Warum es sich lohnt, genauer hinzuschauen. 2. Aufl. Lindenberg i. A. 2021, 96-200.

3 GLA 69 Stürmer Nr. 4 und 7.

4 Siehe die Beiträge von Dagmar Lieske und Hans Günter Hockerts in diesem Band.

5 Die schwierige Frage, ob im vorliegenden Fall tatsächlich der Straftatbestand „sexuellen Missbrauchs" vorliegt - in Abgrenzung zu sexueller Verführung, einvernehmlichem Sexualverkehr und (dem kirchlichen Delikt) Zölibatsverstoß - wäre näher zu diskutieren. Es wird darauf verzichtet, weil Verf. davon ausgeht, dass die „Mechanismen“ des innerkirchlichen Umgangs damit - zumindest was den Täter betrifft - ähnliche waren. 


\section{Der Täter}

Der Lebenslauf des Priesters Karl Lorch (1893-1964), Sohn eines Land- und Gastwirts in Killer, einem kleinen Weiler im Hohenzollerischen, weist bis zu seinem "Fehltritt" - das ist die Bezeichnung, die später in den kirchlichen Akten immer wieder gebraucht wird, und damit ein einmaliges Scheitern unterstellt - keine Auffälligkeiten auf, ist vielmehr typisch zu nennen: Besuch der Volksschule (1899-1904), privater Lateinunterricht beim Ortspfarrer, Zögling des Erzbischöflichen Fideliskonvikts und des Gymnasiums in Sigmaringen (1904-1911), Theologenkonvikt und Universitätsstudium in Freiburg, Priesterweihe (1915, wegen bestehender Gefahr einer Einberufung zum Kriegsdienst möglicherweise in übergroßer Eile), als Vikar pastorale Lehrjahre an verschiedenen Orten (1915 Klosterwald, 1916/17 Trochtelfingen, 1917/18 Lottstetten), dann Kooperator (Kaplan) an St. Martin in Freiburg (1920). Alle seine Zeugnisse - auch die späteren, auch die seiner Kritiker - erkannten einmütig seine intellektuellen und pastoralpraktischen Fähigkeiten an, seine große Arbeitskraft, das positive Echo seiner Predigten in den Gemeinden.

Sein „Fehltritt“ aber riss Lorch 1925 aus den Bahnen des Üblichen. Die Aussicht auf Übernahme einer Pfarrei oder Lehrstelle war mit einem Schlag vernichtet, Lorch wurde fortan nur noch als unselbständiger Geistlicher weiterverwendet und damit - so jedenfalls die Intention des Erzbischöflichen Ordinariats - einer engeren Aufsicht unterstellt. Eine Maßnahme, die allerdings ganz offenkundig nicht ausreichte. Weitere Zölibatsbrüche folgten, die allerdings nie zweifelsfrei nachgewiesen werden konnten, wohl weil es sich um einvernehmliche sexuelle Beziehungen zu Frauen handelte, die eine Aussage verweigerten. 1933 wurde Lorch suspendiert. Nur noch ab und zu konnte er in seiner Heimat einen Gottesdienst übernehmen. 1939 verschwand er quasi über Nacht. Lorch hatte, wie sich später herausstellte, eine Stelle bei der Gauamtsleitung der NS-Volkswohlfahrt in Darmstadt angenommen und den nationalsozialistischen Erwartungen entsprechend dort angegeben, "gottgläubig “6 (also konfessionell ungebunden) zu sein. ${ }^{7}$ Damit schied Lorch freilich faktisch aus dem Priesteramt, auch wenn er gegenüber seinem Erzbischof, der ihn erst aufspüren musste, behauptete, seinen priesterlichen Pflichten

6 So das Ergebnis entsprechender Nachforschungen beim Hauptmeldeamt. Vgl. Ordinariat Mainz an Ordinariat Freiburg, 15.5.1939. EAF Personalakte (PA) Lorch.

7 Ob dies nur den Erwartungen des neuen Brotgebers geschuldet war oder ob hinter dem äußeren biographischen Bruch auch eine innere Abkehr vom christlich-kirchlichen Glauben stand, muss offenbleiben. 
weiter nachkommen zu wollen. ${ }^{8}$ Diesem blieb nur übrig, den Schritt nachträglich zu akzeptieren, wenn auch „unter der Bedingung [...], daß Sie sich Ihres unauslöschlichen priesterlichen Charakters und der daraus sich ergebenden sittlichen Forderungen bewußt bleiben und mir damit eine neue Enttäuschung ersparen“. Einspruch erhob Gröber allerdings gegen den von Lorch erhobenen Vorwurf „einer fast 14-jährigen unchristlichen, unbarmherzigen, unversöhnlich-lieblosen Behandlung" durch das Ordinariat. Davon könne keine Rede sein. Man habe immer wieder erwogen, ihm eine Stelle zu geben. „Wenn es nicht geschah, so wissen Sie, bei wem die Schuld liegt“.

Die ausgesprochene Erwartung, Lorch werde "gläubig und sittlich stark“ sein und „dadurch vielleicht auch eine spätere Verwendung im Kirchendienste selber ermöglichen und anbahnen“9, erfüllte sich nicht. Nur indirekt erfuhr das Ordinariat 1942 von einer bevorstehenden Verheiratung ${ }^{10}$, die dann 1944 erfolgte. Lorch zog sich damit die Tatstrafe der kirchlichen Exkommunikation zu. ${ }^{11}$ Seine Frau erklärte nach seinem Tod 1964 freilich, ihr Mann habe nie mit dem Glauben gebrochen, sei stets zur Kirche gegangen, habe auch seinen „Kirchenbeitrag“ zum Wiederaufbau von St. Ludwig in Darmstadt geleistet und über alle Jahre hinweg Seelenmessen für seine Eltern bestellt. ${ }^{12}$

\section{Zur Quellenlage}

Im Erzbischöflichen Archiv Freiburg liegt eine umfangreiche Personalakte vor, die es erlaubt, das spätere Agieren des Ordinariats relativ genau zu verfolgen. ${ }^{13}$ Allerdings klafft zwischen April 1924 und Juni 1926 eine auffallende Lücke, weshalb sich der Fall selbst anhand der Personalakte nicht mehr rekonstruieren

8 Nicht ohne Vorwurf war der Hinweis in einer Erklärung nach Freiburg, dass ihm „nunmehr von staatlich-weltlicher Seite Stelle und Beschäftigung übertragen wurde“, worum er seit Jahren vergebens das Ordinariat gebeten habe. Lorch, Darmstadt, an Ordinariat, 15.3.1939. Später verwies er darauf, dass seine Anstellung als ,hauptamtlicher Mitarbeiter der Gauamtsleitung der NSV Hessen-Nassau' nichts anderes sei als die Beschäftigung von Priestern bei den Diözesan-Caritasverbänden. Lorch, Darmstadt, an Ordinariat, 7.6.1939. Hier und künftig, sofern nicht anders vermerkt: EAF Personalakte (PA) Lorch.

$9 \quad$ Gröber an Lorch, 10.6.1939.

10 Eine Nachfrage in St. Ludwig, Darmstadt, ergab zunächst nur die Information: „noch nicht verheiratet. Kam am 1.4.1939 von Killer, wohnt jetzt: Darmstadt Schwanenstr. 37 bei Wintting, Witwe, deren Tochter er wahrscheinlich heiratet“, o.D., notiert auf: Ordinariat Freiburg an Pfarramt St. Ludwig, 20.7.1942.

11 Eine entsprechende Feststellungsurkunde o.ä. hat sich nicht erhalten.

12 Geistlicher Rat Meier, Gammertingen, an Erzbischof, 9.1.1965.

13 EAF PA Lorch. 
lässt. Die Lücke ist allerdings nicht, wie vermutet werden könnte, auf eine bewusste Bereinigung durch das Erzbischöfliche Ordinariat zurückzuführen, also auf einen Akt der Vertuschung. Vielmehr mussten die Akten 1942 der Gestapo ausgeliefert werden und kehrten nicht wieder zurück. ${ }^{14}$ Angeblich lag eine Strafanklage gegen Lorch vor; vielleicht war eine propagandistische Ausschlachtung geplant. Für die Rekonstruktion ergibt sich damit das zentrale Problem, dass wir über keine originären Quellen verfügen. Aus der Personalakte lässt sich nicht einmal der Name der Betroffenen ersehen, nicht wie der Fall ans Licht kam, nicht wie die Beteiligten agierten. Die späteren Schriftstücke der Akte (Eingaben Lorchs, Reaktionen der kirchlichen Behörde) lassen nur ausgesprochen dürftige Rückblicke auf den Fall selbst zu.

Dass überihn dennoch berichtetwerden kann, verdanktsich derErmittlungstätigkeit der badischen Gestapo, die sich in Akten niedergeschlagen hat, die deren Leiter Karl Berckmüller (1895-1961) ${ }^{15} 1937$ an den Stürmer, das politpornographische Hetzblatt des mittelfränkischen Gauleiters Julius Streicher $\left(1885^{-1946}\right)^{16}$, übermittelte. Ziel war nicht allein die Diffamierung der Kirche und der Priester insgesamt. Vielmehr sollte Erzbischof Gröber unter Druck gesetzt werden. ${ }^{17}$

In den Fokus der Ermittlungen der Gestapo war schon 1933 das 1924 von Lorch missbrauchte Mädchen geraten, weil man in Kreisen der NSDAP ein sexuelles Beziehungsverhältnis auch zwischen ihr und dem Erzbischof konstruieren zu können glaubte. In diesem Rahmen wurde 1937 der „Fall Lorch“ mitaufgerollt. ${ }^{18}$ Erst aus den Gestapoakten erfahren wir von der Identität

14 Vgl. Ordinariat Freiburg an Pfarramt St. Ludwig, 20.7.1942: „Anscheinend schwebt z. Zt. gegen Lorch ein disziplinäres oder gerichtliches Verfahren; im April dieses Jahres wurden seitens der Geheimen Staatspolizei Freiburg die Personalakten Lorch bei uns erbeten“. Und: „Wir ersuchen um gefällige Mitteilung, bis wann wir der Rückgabe der am 15. April mitgeteilten Personalakten des obigen entgegen sehen können“. Ordinariat Freiburg an Außenstelle der Gestapo in Freiburg, 20.7.1942. - Der Antwort lässt sich entnehmen, dass die Akten "nach Mitteilung der Staatsanwaltschaft Trier" noch benötigt wurden. Gestapo Freiburg an Ordinariat, 27.7.1942. Die Suche nach diesen Akten blieb bislang erfolglos.

$15 \mathrm{Zu}$ ihm: Michael Stolle, Die Geheime Staatspolizei in Baden. Personal, Organisation, Wirkung und Nachwirken einer regionalen Verfolgungsbehörde im Dritten Reich. Konstanz 2001.

16 Zu ihm: Franco Ruault, „Neuschöpfer des deutschen Volkes“. Julius Streicher im Kampf gegen „Rassenschande“. Frankfurt a. M. 20o6; Daniel Roos, Julius Streicher und „Der Stürmer" 1923-1945. Paderborn 2014.

17 Dazu vgl. Burkard, „Dossier Gröber“, 175-184.

18 GLA 69 Stürmer 4. 
der Betroffenen. Ihr Name führte schließlich sowohl zu weiteren staatlichen Akten ${ }^{19}$ als auch zu ergänzenden Akten im Nachlass Gröbers. ${ }^{20}$

\section{Die Betroffene}

Soweit aus all diesen - sehr unterschiedlichen - Überlieferungsschichten (und unter Vorbehalt) rekonstruierbar, wuchs Ottilie G. (1904-1942) ${ }^{21}$ nach dem frühen Tod der Mutter zusammen mit zwei Geschwistern auf. Ihr Vater, ein Freiburger "Stadtarbeiter", ging eine zweite Ehe mit der Schwester der Mutter ein. Nach dem achtjährigen Besuch der Volksschule kam Ottilie G. ein halbes Jahr auf die Frauenarbeitsschule und war anschließend für drei Monate als Zimmermädchen in Stellung. Von Juli 1921 bis Juli 1922 besuchte sie das Freiburger St. Hedwigsheim, wo sie als Hausschülerin zur Kinderpflegerin ausgebildet wurde. Im Jahr darauf erhielt sie - nun als Externe - im St. Hedwigsheim eine Ausbildung zur Säuglingspflegerin, die im November 1923 in der Freiburger Frauenklinik fortgesetzt wurde, bevor sie im Dezember 1923 ins Wöchnerinnenheim nach Konstanz kam. So jedenfalls liest sich die spätere Darstellung Ottilie G.s. ${ }^{22}$ während Gröber, ebenfalls im Rückblick, anders akzentuierte: „Schulentlassen, kam sie später als eine Art Pflegerin ohne Ausbildung in die Klinik, wo sie von den Ärzten als ,blonde Gefahr bezeichnet wurde. Weil sie gefährdet war, kam sie nach Konstanz zu Sr. Maria Elisabeth ins Wöchnerinnen-Heim zur Überwachung". ${ }^{23}$ Von dieser Oberin wurde Gröber als damaliger Konstanzer Stadtpfarrer in seiner Eigenschaft als

19 Im Zusammenhang mit ihrer Zwangssterilisierung. StAF G 1167/1 Nr. 773.

$20 \quad$ EAF Nb 8/140.

21 Der Name wird hier, wie im Folgenden (auch in Zitaten und Archivalienbezeichnungen), als „G.“ abgekürzt.

22 DerLebenslauf nach Ottilie G.s Angaben imVerhörprotokoll:LandeskriminalpolizeistelleGeheime Staatspolizei - bei der Polizeidirektion Freiburg i. Br.: Sittliches Verhalten des kath. Geistlichen Kaplan Lorch, früher Freiburg i. Br., mit Ottilie G., Freiburg, 20.7.1936. GLA 69 Stürmer 4.

23 Gröber: Ottilie G. (2 S., ms), o.D. [nach 1942]. EAF Nb 8/140. - Ähnlich in einer offenkundig früheren Notiz: „Schon als 15-jährige ,die blonde Gefahr'. Mutter früh an Nierenleiden +. Gute Stiefmutter. 16 war von Freiburg weg nach Konstanz ins Wöchnerinnenheim unter die Obhut der Schwester Maria Elisabeth. Von dort nach Freiburg, wo sie ein Verhältnis mit Kooperator von St. Martin Lorch anknüpfte“. Gröber: Charakteristik der G. (hs), o.D. EAF Nb 8/140. 
Beirat des Katholischen Fürsorgevereins für gefährdete und gefallene Frauen und Mädchen hinzugezogen. ${ }^{24}$

Bis zu einer Blinddarmerkrankung im Sommer 1924 war Ottilie G. in Konstanz tätig. Im Wöchnerinnenheim auch operiert, hielt sie sich zur Genesung im Sommer 1924 bei ihren Eltern in Freiburg auf, wo sie die Bekanntschaft mit Kaplan Lorch machte. Nach Konstanz zurückgekehrt, fiel auf, dass sie fast täglich Briefe aus Freiburg erhielt. Von der Oberin des Wöchnerinnenheims zur Rede gestellt, gab Ottilie G. ausweichende Antworten. Der Gestapo gegenüber erklärte sie später, von der Schwester zum damaligen Stadtpfarrer Gröber geschickt worden zu sein, der von ihr die Herausgabe der Briefe forderte. „Er wollte nun wissen, wer der Briefschreiber ist, jedoch nannte ich ihn nicht. Als ich so hartnäckig blieb, sagte er zu mir, gell es ist ein Geistlicher, worauf ich mit Ja antwortete, jedoch den Namen nicht nannte. Hierauf sagte er, den Satan möchte ich kennen, der mich verführt habe. Da ich den Namen nicht nannte, entließ er mich. Durch meine Beichten wusste er aber, dass ich schon Verkehr hatte, denn dieses habe ich ihm gebeichtet. Nach etwa 6-8 Wochen brannte ich im Wöchnerinnenheim durch, weil mir Vikar Lorch immer schrieb, ich solle nach Freiburg zurückkommen“. ${ }^{25}$

Die etwa ein Jahr dauernde Beziehung zwischen Ottilie G. und Lorch hatte Folgen. Ottilie G. wurde schwanger und brachte ein Kind zur Welt, das allerdings wenige Wochen später starb. Die Beziehung zu Lorch wurde, nachdem Ottilie G. seinen Namen preisgegeben hatte, durch das Ordinariat beendet. Danach kam Ottilie G. wieder nach Konstanz, arbeitete als Kinderpflegerin, ging 1926 eine Beziehung mit dem Technikstudenten S. ein. 1927 brachte sie in Essen ein Kind zur Welt. Es folgte 1929 die Heirat und 1930 Geburt eines weiteren Kindes. Das Paar wohnte für kurze Zeit in Chemnitz, aber die Ehe zerfiel, die Beziehung wurde 1931 gelöst. Ottilie G. kehrte nach Freiburg zurück, wo sie sich - nach den Worten Gröbers - „herumtrieb“ aber „immer wieder auf den rechten Weg gebracht werden" konnte. 1934 wurde die Ehe geschieden, wobei das Verschweigen des früheren Verhältnisses sowie Ehebruch mit verschiedenen Männern geltend gemacht wurde. ${ }^{26}$ Die beiden Kinder wuchsen danach bei den Großeltern in Freiburg auf.

24 Gröber, Freiburg, an Kardinalstaatssekretär (Durchschlag, ms), 17.1.1947 [1938!]. EAF Nb 8/140. - Gröbers Auskunft, Ottilie G. sei 1923 „etwa 15 Jahre alt“ gewesen, ist falsch, sie war damals bereits 19, was möglicherweise auf eine noch kindlich-pubertäre Ausstrahlung hinweist.

25 Landeskriminalpolizeistelle, Sittliches Verhalten, 20.7.1936.

26 Offenbar wurde die Ehe auch kirchlich für ungültig erklärt. 
Schon zuvor begann - nach Darstellung Gröbers - mit dem sittlichen auch der psychische Niedergang Ottilie G.s. ${ }^{27} 1932$ begann sie ein Verhältnis mit einem verheirateten städtischen Verwaltungsangestellten St. in Freiburg, das bis 1936 dauerte. Im Herbst 1935 kam Ottilie G. ins Fürsorgeheim nach Singen, wo sie im Dezember ein weiteres Kind zur Welt brachte, ${ }^{28}$ nachdem auf Druck des Vaters offenbar Abtreibungsversuche vorgenommen worden waren. ${ }^{29}$ Das Kind lebte nach der Entbindung bei Ottilie G. in Freiburg, die im Mai 1936 eine Stelle als Fabrikarbeiterin in der Rhodiaseta annahm. 1938 wurde sie nach einem psychischen Zusammenbruch u.a. wegen „reaktiven Depressionen und Degastierungszustandes mit starker vegetativer Beteiligung" ins Lorettokrankenhaus aufgenommen. ${ }^{30}$ Dort beschuldigte sie Ärzte und eine Schwester unsittlicher Handlungen und kam eine Zeit lang in die psychiatrische Klinik. Wieder entlassen, begann sie ein Verhältnis mit einem verheirateten Angestellten der Krankenkasse, das zu einer erneuten Schwangerschaft führte. In der Folge wurde Ottilie G. wegen Abtreibung zu sieben Monaten Gefängnis verurteilt und sterilisiert. Eine weitere Heirat ging in die Brüche. $1942 \mathrm{nahm}$ Ottilie G. sich das Leben. ${ }^{31}$

27 Undatierte, nur stichwortartige Notizen Gröbers deuten eine größere Zahl von Beziehungen an: „Mit Steglitz Schlossberg. Nymphomanin in Endringen I. Selbstmordversuch (Oxalsäure). Fängt mit jungem Mann in Stühlingen an. Verliert meinen Schutz. Fällt mit dem jungen Mann. II. Selbstmordabsicht. [...] Rückkehr des Mannes. Krach mit ihm. III. Selbstmordabsicht. [...] Beicht u[nd] Komm[union] Weihn[achten] 1931. Später Flirt in Stühlingen. Verhältnis in St. Blasien. Kommt nach Konstanz - Reichenau. Not der Familie. Mann arbeitslos. Ehescheidung [...]. Trennung vom Mann. Verschiedene Verhältnisse. Öfters krank und in der Klinik. Diagnose? Bandelt mit Kooperator Hahn an. Zuletzt mit St. Verlangt, dass St. sich scheiden lasse. [...] Abtreibung und zahllose Ehebrüche auch mit andern. Beichtet. Fällt. Kommuniziert ohne Beichte. Kuppelei. Gilt als Dirne, die überwacht wird“. Aktennotizen Gröber zu G. (hs), o.D. EAF Nb 8/140.

28 Gröber: Darstellung des Falles G. (ms, mit hs Ergänzungen), 10.6.1936. EAF Nb 8/140.

29 Damit im Zusammenhang evtl. ein Disziplinarverfahren gegen St., der daraufhin seine Stelle bei der Stadt verlor. Die Familie versuchte, über Gröber eine Wiederanstellung zu erreichen. Sr. Bernarda Bader O.S.B.: Aktennotiz (ms), 2.11.1936. EAF Nb 8/137 Untermappe „Allgemeine Angriffe (Nazi)“.

$30 \quad$ Nervenarzt Dr. Grünewald: Mitteilung (Abschrift), 9.7.1938. EAF Nb 8/140.

31 So die nicht wertfreie Darstellung Gröbers. Vgl. Gröber: Ottilie G. (ms), o.D. [nach 1942]. EAF Nb 8/140. - Die staatlichen Akten zur Sterilisierung in StAF G 1167/1 Nr. 773. 


\section{Der Fall}

Wie gesagt: Erst das mit pikantesten Details gespickte Verhörprotokoll aus dem Jahr 1936, das einzig den „Fall Lorch“ im Jahr 1924 zum Gegenstand hat, ${ }^{32}$ verleiht der Betroffenen eine Stimme. Inwieweit dieses unter Pressionen zustande gekommene, von der Gestapo in erkenntnisleitendem Interesse formulierte „Protokoll“ - im zeitlichen Abstand von 12 Jahren zum Tathergang - der Wahrheit entspricht, lässt sich nachträglich nicht klären.

Demnach lernte die damals 20-Jährige jedoch im Sommer 1924 während ihrer Rekonvaleszenz in ihrer Heimat Lorch zunächst „nur aus der Ferne“ kennen. Als sie ihn beim Beichten - angeblich weil sie einen Klostereintritt erwog - um Rat fragte, bestellte er sie anderntags auf sein Zimmer (aber nicht über den Hauptaufgang, „damit es der Stadtpfarrer nicht sehe“). Dort wurde sie Opfer einer Vergewaltigung. Ottilie G. schildert detailliert ihr erfolgloses, mehrfaches Sich-Sträuben, die Anwendung von Gewalt, das Zufügen von Schmerzen. Obwohl sie „keinen Trieb zum Geschlechtsverkehr verspürte“, kam sie von nun an täglich wieder. Lorch verpflichtete sie zum Stillschweigen. Es ging eine Veränderung mit ihr vor, die in ihrem näheren Umfeld bemerkt wurde, über deren Grund sie sich jedoch ausschwieg. Die Gestapo protokolliert gemeinsame sexuelle Eskapaden, das Bemühen Lorchs, eine Schwangerschaft zu vermeiden, seinen Versuch, eine Abtreibung einzuleiten.

Während sich Ottilie G. weigerte, ihrer Mutter zu sagen, von wem sie schwanger war, eröffnete sie sich einer ihr offenbar von früher her bekannten Ordensschwester des Hedwigsheims. Diese informierte umgehend Domkapitular Adolf Rösch (1869-1962 $)^{33}$, der Ottilie G. ins Ordinariat vorlud. „Dabei war auch die Schwester Lina anwesend. Er fragte mich nun, ob es wahr sei, was ich der Schwester Lina erzählt habe, was ich bejahte. Hierauf begab ich mich sofort zu Lorch und sagte ihm, dass ich auf dem Ordinariat zugegeben habe, dass ich von ihm schwanger sei. Lorch rannte hierauf wie besessen im Zimmer hin und her und sagte, ob ich verrückt sei, so etwas zuzugeben. Wenn ich dieses auf dem Ordinariat nicht zurücknehme, schieße er mich tot“. Von Lorch überredet, ging Ottilie G. abermals ins Ordinariat, wo sie ihre Aussage zurücknahm. „Als mich Dr. Rösch frug, was er denn glauben solle, sagte ich ihm, er müsse eben jetzt glauben, dass ich Lorch verleumdet habe. Damit war die Sache abgetan. Als ich dem Lorch nun sagte, dass ich die Sache zurückgenommen

32 Landeskriminalpolizeistelle, Sittliches Verhalten, 20.7.1936.

33 Zu ihm: Erwin Gatz, Art. Rösch, in: ders. (Hrsg.), Die Bischöfe der deutschsprachigen Länder $1785 / 1803$ bis 1945. Ein biographisches Lexikon. Berlin 1983, 624; Franz Vetter, Adolf Rösch, in: Freiburger Diözesan-Archiv 89, 1969, 488-495. 
hätte, sagte er, es sei recht, das andere fechte er aus. Er wurde dann auf das Ordinariat vorgeladen und wie er mir nachher sagte, hat er dem Dr. Rösch Grobheiten gemacht, weil dieser so etwas von ihm geglaubt habe“.

Lorch veranlasste Ottilie G., zur Geburt des Kindes nach Tübingen zu gehen. Das Kind kam nicht gesund zu Welt und lag drei Wochen in der Tübinger Klinik, während Ottilie G. nach Freiburg zurückkehrte. In dieser Zeit suchte sie auch Gröber auf, der inzwischen Domkapitular geworden und offenbar von Rösch über das Schicksal seines früheren Pfarrkindes informiert worden war. „Ich ging deshalb zu ihm, um mich bei ihm zu entschuldigen, weil ich im Wöchnerinnenheim in Konstanz, wo ich beschäftigt war, durchgebrannt bin. Bei dieser Gelegenheit kam er auf mein Kind zu sprechen. Als er die Photographie sah, sagte er zu mir, dass ich nun nicht mehr sagen brauche, wer der Vater des Kindes sei, er sehe selbst, wer es ist. Er frug mich nun, ob er die Sache in die Hand nehmen dürfe. Hierauf sagte ich ihm, dass es mir gleich sei, ich würde aber nicht sagen, wer der Vater des Kindes ist. Nach drei Tagen war dann Lorch von Freiburg verschwunden und ich habe seit dieser Zeit nichts mehr von ihm gehört. Ich weiß auch heute noch nicht, wo er sich befindet. Nach dieser Zeit nahm sich das Ordinariat um mein Kind an und ich musste es nach seiner Besserung von der Klinik in Tübingen nach Sigmaringen in das Kinderheim Nazareth bringen. Dort ist es dann nach einigen Monaten gestorben".

Aus diesen Angaben der Betroffenen vor der Gestapo ergibt sich - ohne dass dies allerdings durch Dokumente der Personalakte von Lorch gestützt werden könnte: Die Aufdeckung des ,Missbrauchsfalles' erfolgte auf Anzeige einer Ordensschwester und durch Initiative Gröbers, der schon als Beichtvater Ottilie G.s in Konstanz um deren Beziehung wusste, ohne allerdings den Namen des Mannes zu kennen. Die Reaktion der Kirchenbehörde war scharf und bürokratisch. Die Bindungen zwischen Täter und Betroffener wurden gekappt. Lorch wurde zunächst einmal aus dem Verkehr gezogen.

\section{Systemische Beobachtungen}

Der skizzierte Fall physisch-sexuellen und geistlichen Missbrauchs ${ }^{34}$ lässt trotz einer ausgesprochen dürftigen Quellenlage in Bezug auf den eigentlichen

34 So stellt sich die Lage zumindest nach dem von der Gestapo vorgenommenen Verhör dar. Doch sind selbst diese Schilderungen ambivalent. Einerseits schildert Ottilie G. darin die Zwänge und Drohungen Lorchs, seinen unbeherrschten Sexualtrieb, andererseits scheint sie doch an eine tiefere Liebe Lorchs geglaubt zu haben, erwähnt seine „Fürsorglichkeit“ 
Vorgang - wichtige systemische Beobachtungen zu jenen Fragen zu, die sich uns aus heutiger Perspektive stellen.

\section{V.1 Vertuschung und Aufdeckung}

Zuoberst auf der Liste steht die Frage, inwieweit die kirchliche Seite bestrebt war, den Fall zu vertuschen oder zu ignorieren. Anders gewendet: Wurden überhaupt Anstrengungen unternommen, um dem Tun des Kaplans Einhalt zu gebieten und den Wiederholungsfall auszuschließen. Der Befund ist ambivalent; auch muss zwischen den verschiedenen Akteuren unterschieden werden.

a) Verständlicherweise gab sich Lorch - mit Erfolg - größte Mühe, sein Verhältnis zu Ottilie G. geheimzuhalten. Ihre Besuche im Pfarrhaus wurden in Zeiten gelegt, zu denen Pfarrer und Mitkapläne außer Haus waren. Vereinbart wurde der Zugang durch den Hintereingang, außerdem eine Lüge für den Fall der Entdeckung. Man unternahm eine gemeinsame Reise, bei der Ottilie G. als Schwester ausgegeben wurde. Lorch zwang Ottilie G. durch Drohungen zum Stillschweigen bzw. zur Lüge. Er selbst belog auch Ottilie G.s Mutter auf deren besorgte Fragen nach ihrer Wesensveränderung. In den letzten Monaten der Schwangerschaft wurde Ottilie G. auswärts untergebracht. Die Geburt durfte nicht in Freiburg oder überhaupt in Baden stattfinden, sondern im württembergischen,Ausland', im evangelischen Tübingen.

b) Das Bemühen des Konstanzer Wöchnerinnenheims, die offenbar schon zuvor ,Gefährdete - falls dies nicht nur eine nachträgliche, schuldzuweisende Formulierung sein sollte - zu kontrollieren und damit vor einer ihr unzuträglichen Umgebung zu schützen, war nur bedingt erfolgreich. Zum einen weil sich der Fall in Freiburg ereignete und Ottilie G. sich weder mitteilsam zeigte noch zur Aufklärung beitragen wollte (falsche Auskünfte bzw. Auskunftsverweigerung - ob aus eigenem Antrieb, ob aufgrund psychischer oder geistlicher Machtausübung Lorchs), zum anderen weil sie sich der weiteren Aufsicht schließlich durch Flucht entzog (ihr späteres Bedauern, aus dem Wöchnerinnenheim ,ausgebüchst“ zu sein).

c) Eine Freiburger Ordensschwester, der Ottilie G. sich anvertraute, informierte das Ordinariat. Dieses ließ sich durch Einbestellung Ottilie G.s die Aussage bestätigen, wobei es der kirchlichen Seite offenbar nur um eine Tatsachenfeststellung ging, nicht um die Art der Beziehung zwischen

(er unterstützt Ottilie G. während der Schwangerschaft, bezahlt Miete, Entbindungskosten und Krankenhaus, besucht sie) und zeigt auch (weshalb?) keinerlei negative Emotionen gegen Lorch. 
Ottilie G. und Lorch. Als Ottilie G. ihre Aussage auf Druck Lorchs widerrief, wurde auch dieser einbestellt, leugnete und wurde ausfällig. So konnte aus Mangel an Beweisen nicht eingeschritten werden. Erst Gröber konnte Ottilie G. später das indirekte Eingeständnis entlocken, dass Lorch der Vater ihres Kindes sei, und sorgte für Lorchs Entfernung. Da die Betroffene sich über die näheren Umstände ausschwieg und auch gegenüber dem Ordinariat keine Anklage erhob, musste das Ordinariat wohl von einvernehmlichem Sexualverkehr ausgehen. Dies war offenbar die Lesart selbst bei Gröber - der doch um die schon zuvor prekäre Situation Ottilie G.s wusste -, wenn er von „Verführung“ Ottilie G.s durch Lorch spricht. Eine Anzeige vor einem staatlichen Gericht stand deswegen von Anfang an nicht zur Debatte. Weder Täter und Betroffene noch das Ordinariat waren verständlicherweise an einer Publizität der Causa interessiert, die im September 1925 trotzdem „zur Kenntnis weiterer Kreise“ kam. Die (nicht gerichtliche) Anzeige einer weiteren Frau, die behauptete, von Lorch schwanger gewesen zu sein, ${ }^{35}$ hätte aufhorchen lassen müssen, wurde vom Ordinariat aber nicht weiter verfolgt, weil die Frau als „Hysterikerin“ charakterisiert wurde und ihre Geschichte zu „unglaublich“ klang. Man vermutet eine Trittbrettfahrerin mit „krankhaft-erotischen Phantasien“. ${ }^{36}$

d) Gröber hielt, nachdem er 1932 Erzbischof geworden war, zunächst am Kurs des Ordinariats fest, das sich weigerte, Lorch in der Pastoral des Erzbistums einzusetzen, und stattdessen eine Verwendung a) außerhalb des Erzbistums und b) an einer Stelle, wo er möglichst wenig Schaden anrichten konnte, befürwortete. Ab April 1933 ließ Gröber eine Wiederverwendung Lorchs im Erzbistum - als unselbständiger Geistlicher - zu. Vorangegangen waren zahllose Bittschriften Lorchs, ein ärztliches Attest und Empfehlungsschreiben von dritter Seite. Doch bereits im August 1933 kochte die NS-Presse den Fall wieder auf, ${ }^{37}$ so dass Gröber sich gezwungen sah, Lorch erneut zurückzuziehen. ${ }^{38}$ Dieser zeigte sich bei der Mitteilung seiner Suspendierung durch

35 Kath. Anstaltspfr. der Strafanstalten Bruchsal (Gutfleisch) an Ordinariat, 25.10.1927. - In seinem Besitz befand sich eine Niederschrift der Frau.

36 Ordinariat Freiburg an Gutfleisch, 22.10.1927.

37 Art. Sicher kein Heiliger!, in: Der Alemanne Nr. 224 vom 15.8.1933. - Informant war vermutlich Domkapitular Josef Sester (1877-1938), der damals versuchte, Gröber aus dem Amt zu drängen. Vgl. Burkard, „Dossier Gröber“, insbes. 120-122.

38 Der Erlass vom 17.8.1933 (Nr. 10446) ist in der Personalakte nicht erhalten, jedoch eine Bescheinigung Lorchs über dessen Bekanntgabe: „daß das Hochwürdigste Erzbischöfliche Ordinariat nach der schweren Diffamierung seiner Person in der Öffentlichkeit nicht in der Lage ist, ihn vorerst in der Erzdiözese zu verwenden u. daß der Hochwürdigste Herr Erzbischof ihm mit Rücksicht auf diese öffentliche Bloßstellung die Vornahme öffentlicher kirchlicher Funktionen in der Erzdiözese untersagt. Von einer 
den Dekan „sehr niedergeschlagen und weinte“. ${ }^{39}$ Gröber sah die Ursachen des Falls von 1924 offenbar nicht allein bei Lorch liegen. So erklärte er - in anderem Zusammenhang - Ottilie G. sei „unstreitig schwer erblich belastet", wie ihr ganzes Leben beweise, und fasse „alles sexuell auf“. Worauf Gröber diese freilich exkulpierend gemeinte Aussage stützte (das angedeutete sexuelle ,Vorleben' Ottilie G.s, ihre geistig-psychisch-sozial prekäre Verfassung?) bleibt offen. Allerdings stellte Gröber auch fest, Ottilie G. sei durch Lorch "schon früh schmählich verdorben" worden, ${ }^{40}$ womit er Lorch immerhin eine erhebliche Mitschuld am späteren Geschick Ottilie G.s zuerkannte. Dies sah auch die Gestapo so: „Gerade durch ihre [Ottilie G.s] religiöse Veranlagung sowohl, als auch durch ihre Unerfahrenheit und nicht zuletzt durch den Beichtstuhl dürfte sie in sittlicher Hinsicht auf die schiefe Ebene gekommen sein, denn gerade im Beichtstuhl lernte sie den Pfarrer Lorch kennen, der sie vom Beichtstuhl aus auf sein Zimmer bestellte, sie verführte, ihr die Unschuld raubte und die längere Zeit hindurch in unverantwortlicher Weise geschlechtlich missbrauchte und zwar zum Teil in ekelerregendster Weise, zudem auch noch in einem katholischen Pfarrhaus und ihrem damaligen Alter von kaum 20 Jahren“. ${ }^{41}$ Wenn die Gestapo allerdings davon sprach, dass "gewisse Kreise am Werk" seien, die versuchten, „die ganze Schuld auf die G. abzuladen und sie als sittlich verwahrlost, unglaubwürdig und geistesschwach zu brandmarken“, so sollte damit vor allem Gröbers Position geschwächt werden, der sich damals gegen die Anschuldigung wehren musste, selbst ein sexuelles Verhältnis mit Ottilie G. gehabt zu haben. Das gerichtsärztliche Gutachten, das damals bei Ottilie G. „angeborenen Schwachsinn“ feststellte und zu ihrer Sterilisierung führte, ging freilich nicht auf Gröber zurück, sondern war im Rahmen des von den NS-Behörden verfolgten, von der Kirche aber vehement abgelehnten

eigentlichen Suspendierung ist abgesehen. Er wird alsbald seinen Posten verlassen“. Lorch: Bescheinigung, 18.8.1933.

39 Dekanat Tauberbischofsheim (Gramling) an Ordinariat, 30.8.1933. - Am 2. September wurde Lorch telefonisch ins Priesterhaus nach Neuburg a. D. gewiesen. - Der Artikel hatte ein weiteres Nachspiel: Weil Lorch in einer Zuschrift an den Alemannen - und wohl nicht zu Unrecht - Sester verdächtigte, gegen ihn agiert zu haben, reichte dieser beim Offizialat Strafanzeige ein, woraufhin Lorch ins Priesterhaus Weiterdingen verwiesen wurde. Ordinariat an Lorch, 3.10.1933.

40 Gröber: „Eidesstattliche Erklärung“ (ms mit hs Ergänzungen), 2.7.1936. GLA 69 Stürmer Nr. 4. - Die Gestapo bestätigte, folgerte daraus jedoch: „Wenn daher heute die G. als sittlich verkommen hingestellt wird, so fällt eine wesentliche Schuld auf diesen sittenlosen Geistlichen“. Geheimes Staatspolizeiamt (Berckmüller), Karlsruhe, an Gröber, 24.7.1936. EAF Nb 8/135.

41 Gestapo: Geisteszustand der Ottilie G. (Abschrift), 22.7.1936. GLA 69 Stürmer 4. 
eugenischen Programms des Nationalsozialismus erstattet worden. ${ }^{42}$ Man könnte hier also durchaus von einem gleich mehrfachen Missbrauch Ottilie G.s durch unterschiedliche Akteure reden.

\section{V.2 Verantwortlichkeiten}

Spätestens mit dem Bekanntwerden des Falles in Freiburg stellt sich die Frage nach der Verantwortlichkeit von Erzbischof und Ordinariat, zum einen hinsichtlich der weiteren Behandlung von Lorch, zum anderen hinsichtlich der Frage, ob man es in der Vergangenheit an der notwendigen Sorgfaltspflicht hatte mangeln lassen. Dem Historiker zeigt sich bei einer kritischen Durchsicht der Personalakte von Lorch, dass es eventuell bereits vor 1924 Anzeichen für mögliche Probleme in sexueller Hinsicht gab:

a) Beim Eintritt ins Freiburger Theologenkonvikt motivierte Lorch seinen Entschluss ausdrücklich nicht mit einer sonst üblichen Formel, etwa sich „zum Priestertum berufen“ zu fühlen o.ä., sondern damit, er habe sich "nun nach reiflicher Überlegung entschlossen, zum Studium der Theologie überzugehen“. ${ }^{43}$ In dieser unüblichen Formulierung könnten sich bereits - versteckt - Berufszweifel artikulieren, möglicherweise sogar Hinweise auf eine nicht ganz freie Berufsentscheidung. Dazu würde die allerdings erst viel später gemachte Aussage von Lorchs Bruder passen, Karl sei von den Eltern zum Priesterberuf gezwungen worden. ${ }^{44}$

b) 1917 verlangte der Prinzipal Lorchs vom Ordinariat dessen rasche Entfernung: „Es hat sich schon zu viel Freundschaft entwickelt in gewissen Häusern, wo junge Mädchen sind. Er läßt sich einziehen. Von verschiedenen Seiten [...] bin ich darauf aufmerksam gemacht worden. Ich denke, daß noch

Amtsärztliches Gutachten (auf Vordruck): „Diagnose: angeborener Schwachsinn, fortpflanzungsfähig, operationsfähig, kein Kretinismus“, 15·7.1936. StAF G 1167/1 Nr. 773. Abiturient Lorch: Curriculum Vitae, o.D. [1911].

44 „Wie wir erfahren, [...] soll der Bruder Lorch's geäußert haben, Karl sei durch seinen Vater mit schweren Drohungen gezwungen worden, den geistlichen Beruf zu ergreifen. Wir haben eher Gegenteiliges von dem Vater gehört, der als wenig kirchlicher Gesinnung geschildert wurde, dem es ein Opfer war, seinen begabten Sohn der Kirche zu schenken“. Ordinariat an Pfr. Diewald in Hausen i. K., 14.8.1947. - Demgegenüber berichtete der Heimatpfarrer Lorchs: „Sein Vater besaß eine kirchentreue Gesinnung und hat Sonntag für Sonntag seine religiösen Pflichten erfüllt. Die Orientierung des Hochw. Erzb. Ordinariates über die Gesinnung des Vaters dürfte irrig sein. Die angeschlossene Erklärung des Bruders Michael Lorch bezeugt, daß ein Zwang des Vaters bei der Berufswahl vorgelegen hat". Pfarramt Hausen i. K. (Diewald) an Ordinariat, 24.8.1947. - Weitere Personen bestätigen den Eindruck eines vor der Weihe vorliegenden Zwanges oder zumindest einer bestehenden Furcht. In diesem Zusammenhang ist auch von einer spätpubertären Entwicklungsverzögerung bei Lorch die Rede. 
nichts vorgekommen ist, aber mit der Zeit könnte es gefährlich werden, weil es schon lange währt. Ich will nicht, daß für den Vikar Schaden entstehe und sich nach und nach Schlimmes entwickelt" ${ }^{45}$ Auf Nachfragen erläuterte der Pfarrer: „Herr Bürgermeister hat mir im Vertrauen auf dem Rathaus mitgeteilt: ich möchte den H. Vikar warnen, nicht mehr so oft in ein Haus zu gehen, das er für gut hält, aber nicht in diesem Rufe steht, um in nichts hineinzukommen; er werde es nicht besser wissen; ebenso in ein anderes, wo man den Vikar auch einzieht; damit kein Anstoß erregt wird“. Allerdings glaubte der Pfarrer wenig später Entwarnung geben zu können, „[...] indem die Personen, um die es sich handelt, teils verzogen sind, teils sich verheiratet haben“. ${ }^{46}$

c) 1924 - zu einer Zeit, in der der ,Fall' bereits bestand - bemerkte auch der Freiburger Prinzipal gegenüber dem Ordinariat, Lorch habe sich "gegen den Ansturm der Frauenwelt besonders zu wehren" ${ }^{47}$ Im gleichen Bericht heißt es über seine Betätigung in der außerordentlichen Seelsorge, Lorch sei in der Klinik eingesetzt, wo er auch Vorträge für die Schwestern halte und Hebammenunterricht gebe. Ein tatsächliches Problem wird vom Pfarrer (damals) nicht gesehen - im Nachhinein aber lässt diese Mitteilung aufhorchen, zumal Lorch im Fall Ottilie G. ausgerechnet Kontakt zu einem in der Säuglingspflege ausgebildeten Mädchen (ob dem einzigen?) aufgenommen hatte.

Diese Hinweise aus der Personalakte lassen sich nachträglich als Indizien lesen, die die Verantwortlichen zu einer gewissen Vorsicht hätten bringen können, wobei es sich bei a) um ein eher schwaches, bei b) und c) um stärkere Indizien handelte. Allerdings: Die Pfarrer scheuten sich offenbar, entsprechende Beobachtungen aktenkundig zu machen und so - möglicherweise ja auch grundlos - einerseits einen jungen Mitbruder bei der Behörde in Misskredit zu bringen, andererseits das nötige Vertrauensverhältnis und damit auch die tägliche Zusammenarbeit zu belasten. Lediglich einer der Prinzipale zeigte die Gefährdung und/oder Gefährlichkeit Lorchs an, wenn auch nur in einem privaten Schreiben an ein Mitglied des Ordinariats und „im Vertrauen“ auf dessen „strengste Verschwiegenheit“; er wolle nicht, dass die Sache „beim Ordinariat oder sonstwo zur Sprache kommt und der Herr Vikar dadurch in Schaden kommt; es ist ja noch nichts Schlimmes geschehen“. Aber er sagte auch deutlich, er wolle den Abzug des Vikars und damit seine „Verantwortung los" sein. ${ }^{48} \mathrm{Ob}$ seine „Entwarnung“ wenig später gerechtfertigt war? Oder hatte er nur ,kalte Füße bekommen („es ist nun alles wieder in Ordnung; es braucht

48 Pfr. Kech, Trochtelfingen, an Assessor/Geistlichen Rat [Rösch], 5·9.1917. 
keiner verwarnenden Mitteilung an den Vikar mehr")? Einen Wechsel hielt er auch ferner für sehr erwünscht. ${ }^{49} \mathrm{Im}$ Dienstzeugnis war dann aber nicht mehr der leiseste Anklang diesbezüglicher Zweifel zu vernehmen. ${ }^{50}$

Das Ordinariat ging mit den Indizien vor dem ,Missbrauchsfall‘ zurückhaltend um. Lorch wurde aber - trotz der „Entwarnung“ seines Prinzipals - rasch versetzt. Auch verweigerte man sich ziemlich kategorisch dem Antrag Lorchs auf Übertragung einer Religionslehrerstelle an der Rotteck-Oberrealschule, vielleicht weil man ihn gerade nicht mit Jugendlichen zusammenbringen wollte. ${ }^{51}$

\section{V.3 Wiederverwendung des Täters}

Wie wir sahen, reagierte das Ordinariat (Gröber) nach der offenkundigen Schuld von Lorch rasch und entschieden. Allerdings bewegte sich diese Reaktion ganz im Rahmen des eigenen Systems. Es kam zu keiner gerichtlichen

49 Denn: „neben einem so eingebildeten Vikar, der alles besser wissen will, nach dem kein Geistlicher der Erzdiözese etwas ist und nach dem alle meine Vikare vorher nichts waren, hält man es fast nicht aus“. Pfr. Kech, Trochtelfingen, an Assessor/Geistlichen Rat [Rösch], 13.9.1917.

$5^{\circ}$ "Sein religiöses Leben war priesterlich; sein sittlicher Wandel tadellos; sein Benehmen außer dem Hause war, wie es recht ist; überaus gefällig, freundlich und liebenswürdig, im Hause war es grundlos anders. Lorch ist gesellschaftlich veranlagt; sein Eifer im Studium ist groß; [...] Form, Inhalt, Vortrag, Erfolg seiner Predigt war gut. Seine Schulkinder hielt er in Kirche und Schule in strengem Zügel und zeigte Geschick und Eifer im Unterricht; er war ein stets bereiter Krankenseelsorger [...]“. Pfr. Kech, Trochtelfingen, an Ordinariat, [24.10.1917]. - Tatsächlich wurde Lorch auch auf seiner nächsten Stelle wieder das beste Zeugnis ausgestellt: „Vikar Lorch ist von ausgeprägt selbständigem Charakter; was sein religiöses Leben betrifft, [...] befliß sich eines guten sittlichen Wandels; was den Verkehr betrifft, so war er gern für sich allein, seinen Studien ergeben, ging wenig aus, wusste sich aber bei gegebener Gelegenheit gut mit anderen zu unterhalten“. Pfarramt Lottstetten (Wachter) an Ordinariat, 1.6.1918.

51 Es fällt auf, dass Loch ausgerechnet 1924 versuchte, der engen Kontrolle im Pfarrhaus zu entkommen und als Religionslehrer nicht nur eine größere Freiheit, sondern auch eine „natürliche“ Möglichkeit des Umgangs mit jungen Menschen zu erhalten. In diese Richtung scheint auch seine idealisierte Motivation zu weisen: Freunde und eigene Erfahrungen hätten ihn in seiner Überzeugung bestärkt, „wie wichtig und verantwortungsvoll der Religionsunterricht an einer Mittelschule heutzutage ist, daß er nicht bloß ein Unterrichtsfach, wie andere, sein darf, sondern daß er das wesentliche Stück der Herzens- und Charakterbildung, vor allem Seelsorge sein muß, mit ganzer Hingebung, die den Schülern auch außerhalb des Unterrichts nachzugehen und persönlich auf sie einzuwirken bestrebt ist". Lorch, Freiburg, an Ordinariat, 8.4.1924. - Vielleicht wollte man genau dies nicht. Das Ordinariat erwiderte am 24.4.1924, für die gewünschte Stelle lägen bereits anderweitige Vorschläge im Ministerium. Eine Beurlaubung zu theologischen Studien unter kirchlicher Sustentation sei nicht machbar und auch sonst unmöglich. Man könne lediglich die Versetzung auf eine weniger arbeitsreiche Stelle erwägen. Ebd. 
Anzeige, was freilich dem expliziten Willen der Betroffenen entsprach. Kirchlicherseits scheint die Ahndung des Vergehens auf dem reinen Verwaltungsweg geschehen zu sein, also auch ohne ein kirchengerichtliches Verfahren. Auf Anordnung des Ordinariats wurde Lorch zunächst isoliert und in ein Kloster eingewiesen. Mit dem Entzug der Stelle verlor er sein Gehalt, verursachte andererseits dem Ordinariat aber erhebliche Kosten. Für Lorch hatte die fehlende Anstellung finanzielle Auswirkungen, an deren Folgen Aufhäufung eines Schuldenbergs - er über Jahre hinweg zu tragen hatte. Der Aufenthalt im Kloster hatte einen mehrfachen Zweck: Er sollte Lorch und Ottilie G. zunächst einmal trennen, sodann der inneren ,Sortierung dienen, war für Lorch zudem als Bußzeit und Strafe gedacht.

Von Anfang an stand freilich die drängende Frage nach der weiteren Verwendung Lorchs im Raum. Die Behandlung gerade dieser Frage lässt sich aufgrund einer exorbitanten Quellendichte in dieser Hinsicht am Fall Lorch eingehend studieren. ${ }^{52}$ Hier kann nur auf Weniges hingewiesen werden:

a) Das Ordinariat vertrat die Maxime, Lorch vorerst nicht mehr (und später: keinesfalls mehr) im Erzbistum anstellen zu können, suchte ihn in einem anderen Bistum oder bei einem Orden unterzubringen. Das eigentliche Movens hierfür lag weniger darin, etwaige künftige Fehltritte Lorchs zu verhindern, als vielmehr in der verdorbenen Fama Lorchs. Mehrfach wird ausdrücklich die schwere „Diffamation“ Lorchs im Erzbistum angesprochen. Die angefragten Diözesen und Klöster ließ das Ordinariat jedoch kein einziges Mal im Unklaren über die Vergangenheit Lorchs. ${ }^{53}$

b) Lorch drängte das Ordinariat in Dutzenden von Petitionen und Eingaben, ihm eine Pastoral- oder eine Lehrerstelle zu übertragen bzw. zu gestatten. Ob er in der Schule einen neuen ,Dunkelraum' suchte? Die Tatsache, dass Lorch sich mehrfach vor allem jüngeren Mädchen näherte, könnte darauf hindeuten. Das Ordinariat untersagte ihm deshalb zu Recht die Annahme einer Stelle an

$5^{2}$ Die PA enthält eine Masse an Briefen von Lorch mit Bitte um Zuweisung einer Stelle sowie mit Rechnungen, die das Ordinariat für seine Unterbringung begleichen musste.

53 So ließ man Rottenburg etwa wissen, Lorch sei „ein sehr befähigter Geistlicher, auch ein guter Redner und Prediger, aber leider von etwas zu weichem Charakter“. Man habe ihn im Spätjahr 1925 „wegen unzüchtigen Verkehrs mit einem hiesigen Mädchen, das nicht ohne Folgen blieb“, von seiner Stelle entfernen müssen, zuletzt habe er (in Sachsen) „ein einwandfreies Zeugnis“ erhalten. Graf Neipperg sei von dem früheren Fehltritt Lorchs unterrichtet. „Das Kind ist gestorben und ist anzunehmen, dass jede Beziehung Lorchs zu dem betreffenden Mädchen aufgehört hat. Da wir Herrn Lorch wegen der schweren Diffamation infolge seines Fehltrittes in unserer Diözese vorerst nicht verwenden können, wären wir Euer Bischöflichen Gnaden dankbar, wenn Sie ihm für die dortige Diözese die erforderlichen Fakultäten verleihen wollten“. Ordinariat Freiburg an Bischof Sproll, 13.9.1927. 
einer Mädchenschule in Euskirchen/Rheinland. ${ }^{54}$ Etliche Klöster lehnten eine Übernahme Lorchs ins Angestelltenverhältnis explizit mit dem Hinweis auf eine Gefahr für die Schüler ab. ${ }^{55}$ Ein Versuch als Hausgeistlicher im Kloster St. Maria in Mönchengladbach scheiterte wegen eines neuen Vorfalls. ${ }^{56}$ Auch sonst gab es beunruhigende Verdachtsmomente, ${ }^{57}$ so dass das Offizialat $193^{2}$ gegen Lorch ein kirchliches Strafverfahren wegen Verletzung seiner Standespflichten (CIC 1917, c. 2359) ${ }^{58}$ eröffnete, in dessen Verlauf weitere Verfehlungen ans Licht kamen. Das Urteil lautete auf Suspension für sechs Monate; außerdem hatte sich Lorch für die Dauer von mindestens einem Monat in einem geistlichen Haus einer „Geisteserneuerung“ zu unterziehen; die empfindlichste Konsequenz war der Verlust seiner Pensionsberechtigung. ${ }^{59}$

d) Die eigentliche Schwierigkeit bestand für das Ordinariat darin, einen Ort für die Verwahrung Lorchs zu finden. Immer nur übergangsweise fand

54 „Zu einer Lehrstelle an einer höheren Mädchenschule können wir Sie aus Gründen, die wir Ihnen nicht auszuführen brauchen, nicht für geeignet erachten. Eine Verwendung an einer höheren Schule könnte für Sie nur an einer Privatschule für Knaben, insbesondere an einer von einem Orden geleiteten, in Frage kommen“. Ordinariat Freiburg an Lorch, 10.10.1930.

55 So erklärte beispielsweise der Abt des Klosters Engelberg im Oktober 1931: „Immerhin werde ich die Frage nicht los, ob es gut sei, einen in diesem Punkte so schwachen Herren zu jungen Leuten zu lassen. Jeder Abt hat eine schwere Verantwortung für die Studierenden, welche von den Eltern dem Kloster zur Erziehung übergeben werden; und wenn dann etwas Unrechtes vorkäme, so würde man nicht nur den angestellten Herrn, sondern auch denjenigen, der ihn anstellte, dafür verantwortlich machen“. Abtei Engelberg an Ordinariat, 20.10.1931.

$5^{6}$ Lorch hatte mit einer jungen Witwe ein „Liebesverhältnis“ begonnen. Im Juli 1931 tauchte er für mehrere Monate unter, ohne dass das Freiburger Ordinariat seinen Aufenthaltsort in Erfahrung bringen konnte.

57 Als „geparkter“ Hausgeistlicher im Altersheim Gamertingen musste Lorch offenbar wegen Annäherungen an Haushaltsschülerinnen entfernt werden. Die Oberin der Schwestern in Gamertingen berichtete dem Ortspfarrer besorgt: „Denken Sie sich, gestern Abend (also Mittwoch) nach dem Abendessen ging H. Lorch um 7 Uhr in die Haushaltungsschule hinab zu den Mädchen \& war dort bis 9 Uhr, bis die Lehrschwester, die ihn anders nicht fortbrachte, bündig erklärte: Ihr Mädchen geht jetzt ins Bett. Dann erst sei er gegangen. Er hätte von der Schwester ein Verzeichnis der Schülerinnen mit Angabe ihres Geburtsortes verlangt. Die Lehrschwester habe sich ausgeredet, sie hätte noch kein Verzeichnis [...] darauf habe Lorch die Mädchen einzeln über alles ausgefragt“". Pfr. Bogenschütz, Trochtelfingen, an Ordinariat, 19.11.1931.

$5^{8}$ Kapitularvikar Sester an Ordinariatsrat und Promotor iustitiae Josef Vögtle, 11.1.1932. Nach Abschluss der Untersuchungen wurde die Anklageschrift im April 1932 durch eine weitere ergänzt.

59 Gemäß $\S 6$ a des Pensionsstatuts für die hohenzoll. Geistlichen vom 15. Dezember 19o9, allerdings unbeschadet des Anspruchs auf den Tischtitel. Urteil des Offizialats Freiburg (Rösch), 28.6.1932. 
Lorch Zuflucht in der im Erzbistum liegenden Benediktinerabtei Beuron, im Priesterhospiz der Barmherzigen Brüder in Neuburg a. D., im Priesterhaus Weiterdingen sowie im Exerzitienhaus der Jesuiten in Feldkirch. Anfragen Beurons bei mehreren süddeutschen Klöstern und Kongregationen mit Privatschulen, um Lorch eine Stellung außerhalb der Pastoral zu bringen, waren ohne Erfolg. Noch im Februar 1933 hielt das Ordinariat - trotz verschiedener positiver Zeugnisse und eines ärztlichen Attests ${ }^{60}$ - die Zeit nicht für gekommen, Lorch im Erzbistum eine neue Anstellung zu geben. ${ }^{61} \mathrm{Im}$ April 1933 unternahm Gröber schließlich einen Versuch mit Lorch als Vikar in Kühlsheim, im äußersten Zipfel der Erzdiözese. Doch machte die von der NSPresse betriebene öffentliche „Diffamation“ gegen Lorch alles zunichte. Später erneut aufgenommene Bemühungen, ihm in anderen Diözesen oder bei einem Orden eine Anstellung zu verschaffen, ${ }^{62}$ blieben ohne Erfolg. Bis Juni 1938 kam Lorch bei den Barmherzigen Brüdern in Neuburg unter, danach hielt er sich ohne Stelle in seiner Heimat Killer auf, von wo aus er gelegentlich Aushilfen im benachbarten Jungingen übernahm, bis er 1939 das Priesteramt aufgab.

\section{Fazit}

Im vorliegenden Beitrag tritt uns das Thema ,Missbrauch' als Teil eines offenbar generellen Zölibatsproblems entgegen, das den Täter in eine zunehmend aussichtslose Lage manövriert. Zugespitzt formuliert: Der (mutmaßliche) Missbrauch eines 20-jährigen Mädchens in prekärer Situation wird - freilich vorausgesetzt, es handelte sich um den ersten sexuellen Übergriff des

6o Der Arzt Lorchs konstatierte einen völligen nervlichen Zusammenbruch sowie „erhebliche Gemütsdepressionen neben einer stark ausgeprägten Herz- und Magenneurose“. Er führte den Zustand „mit Sicherheit“ auf den "Mangel einer geregelten beruflichen Tätigkeit und Jahre dauernde Existenzlosigkeit“ zurück. Lorch sei ,jetzt ein völlig körperlich und vor allem seelisch zusammengebrochener Mensch“. Eine Besserung könne nur erfolgen, „wenn ihm bald wieder ein seinen geistigen und körperlichen Fähigkeiten entsprechendes Arbeitsfeld angewiesen“ werde. Dr. med. Heitzler, Burladingen: Ärztliches Attest, 16.1.1932.

61 Ordinariat Freiburg an Lorch, 16.2.1933. - Lorch hatte an das Ordinariat die „inständige Bitte um Arbeit durch Wiederbeschäftigung“" gerichtet, weil „seine frühere Berufs- und Arbeitsfreude immer mehr durch schwermütige Depressionen gelähmt" werde. Lorch, Neuburg a. D., an Ordinariat, 7.2.1933.

62 Dies glaubte man inzwischen versuchen zu können: „Lorch hat sich unseres Wissens in den letzten Jahren sittlich gut gehalten. Verfehlungen gegen die Strafgesetze des Staates und perverse Neigungen insbesondere gegenüber dem männlichen Geschlecht liegen bei ihm nicht vor". Ordinariat Freiburg (Durchschlag), 26.4.1935. 
Täters - zu einer Zäsur oder Lebenswende. Es folgen in den nächsten Jahren und Jahrzehnten weitere Zölibatsverstöße, allerdings soweit erkennbar nicht mehr mit Minderjährigen, sondern mit erwachsenen (verheirateten, unverheirateten und verwitweten) Frauen. Das Problem ,Missbrauch' erscheint hier also nicht als Pädophilie, selbst nicht in ihrem weitesten Sinne, sondern als unbewältigte Sexualität bzw. als Scheitern an der kirchlich vorgegebenen Norm der zölibatären Lebensform. Der pastorale Kontakt (nicht der Beichtstuhl selbst, aber der - gesuchte - ,Rat'), mithin die moralische Autorität des Geistlichen, ermöglicht den geistlichen Machtmissbrauch, gepaart mit sexuellem Missbrauch.

Ungewöhnlich ist der durch, fremde 'Quellen mögliche Blick der Betroffenen, freilich in der Brechung eines (interessengeleiteten) Gestapo-Protokolls. Dieses lässt das Täterprofil deutlich hervortreten: das Profil eines Menschen, der von extremem Sexualtrieb gesteuert, Gelegenheiten sucht und schafft, der raffiniert und zielgerichtet vorgeht, (zumindest anfänglich vorhandenen) Widerstand erfolgreich bricht und schließlich täglichen Sexualverkehr einfordert, der die Betroffene an sich bindet und (zumindest bis zu der vom Ordinariat erzwungenen Beendigung der Beziehung und seiner räumlichen Entfernung) auch psychisch Macht ausübt, der sich mehrfach "Schweigen“ versprechen lässt, der (erfolglos) versucht, das Beichten der Betroffenen bei einem anderen Priester (Gröber) zu unterbinden, den die Schwangerschaft in Panik versetzt, der auf Abtreibung dringt und diese selbst vorzunehmen versucht. Eines Menschen allerdings auch, der der Betroffenen eine gemeinsame Zukunft (freilich nicht in der Ehe) verspricht und an diese glauben lässt, der sich im Zusammenhang mit der Schwangerschaft zumindest finanziell in die Pflicht nehmen lässt, ${ }^{63}$ von dem man letztlich aber nicht weiß, wie heiß

63 „In diesen Briefen schrieb er meistens von Liebe und auch Schweinereien. [...] Auf der Reise nach Freiburg gab er mir Obst, Trauben, Schokolade und sonstiges mit. Ob er mir auch Geld gab, weiss ich nicht mehr, glaube es aber. [...] Von seiner Heimat aus hat er während seiner Ferien fast täglich geschrieben, und zwar postlagernd. In den Briefen erkundigte er sich nach meinem Ergehen [...]. Von Lorch bekam ich hierzu [Fahrt nach Tübingen] das Fahrgeld und gab mir auch sonst noch Geld zum Leben mit. [...] Solange ich in Tübingen war, wurde ich fortlaufend von Lorch unterstützt. Er bezahlte auch die Entbindungskosten und das Krankenhaus. [...] Als ich nach Freiburg zurückkam, kam ich noch etwa 3 Wochen mit Lorch zusammen. [...] Nach 3 Tagen war dann Lorch von Freiburg verschwunden und ich habe seit dieser Zeit nichts mehr von ihm gehört. Ich weiss auch heute nicht, wo er sich befindet. Nach dieser Zeit nahm sich das Ordinariat um mein Kind an $[\ldots . .$. “. Landeskriminalpolizeistelle, Sittliches Verhalten, 20.7.1936. 
oder kalt ihn das Ergehen der Betroffenen lässt, und der später (ebenfalls) in zunehmend depressive Stimmungen gerät. ${ }^{64}$

Von sich selbst zeichnet die Betroffene im Rückblick das Bild eines naiven, gutgläubigen Mädchens, das ihren Vergewaltiger mit "großen Augen“ ansieht, sich zwar wehrt, aber doch auch nachzugeben scheint - ein Mädchen, das alles glaubt, was ihm versprochen wird, das sich dominieren lässt, die eigene Stellung aufgibt und auch später an einer Beendigung der Beziehung nicht interessiert zu sein scheint. Hier wird die Ambivalenz der Quellen in ihrer Gesamtschau deutlich: Anfänglicher Missbrauch, Vergewaltigung und eine dynamische Entwicklung der Beziehung - möglicherweise in Richtung Einvernehmlichkeit ${ }^{65}$ - scheinen sich nicht per se auszuschließen. Dazu passt die Supposition der Quellen, die nicht nur beim Täter (in der Beschreibung der Betroffenen) einen ausgeprägten Sexualtrieb konstatieren, sondern auch die Betroffene selbst als (später?) stark sexualisiert erscheinen lassen. ${ }^{66}$ Es lassen sich bei der Betroffenen auch Formen von Widerstand erkennen. Meist bleiben sie im Ansatz stecken: Ihr körperliches Sich-Wehren (und Nachgeben), ihre verbalen Einwände (und das Sich-Überredenlassen), das Beichten ihres außerehelichen Geschlechtsverkehrs (und die Weigerung, Namen zu nennen), das Sich-Offenbaren gegenüber einer Ordensschwester (und die Verschlossenheit gegenüber Stiefmutter und Tante), ihre Aussage auf dem Ordinariat (und die Rücknahme dieser Aussage nach dem, Austicken und den Drohungen Lorchs).

Wir haben es mit komplexen Täter- und Opferfiguren zu tun. Dies nicht infolge einer Übermacht kirchlicher Quellen mit einseitigen Schuldzuweisungen, sondern weil die Betroffene selbst widersprüchliche Aussagen über das Verhältnis zu Lorch zu Protokoll gibt, die sie einmal zum ,Opfer - ein andermal zur, Täterin' machen. ${ }^{67}$ Diese unterschiedlichen Aussagen Ottilie G.s wurden im Abstand vieler Jahre, in ganz verschiedenen Lebenssituationen und gegenüber zwar gleichermaßen bedrohlichen, jedoch unterschiedlichen Instanzen (Gestapo, Gerichtsarzt) abgegeben. Nur vermuten lässt sich, dass

64 Das Ordinariat ließ sich - nach weiteren Vorfällen - erst spät durch das fortgesetzte Bitten Lorchs erweichen, seiner „Not“ durch Wiederermöglichung pastoraler Betätigung zu steuern.

65 „Später wurden wir so frech, dass ich auch nachts auf sein Zimmer kam [...]“. Landeskriminalpolizeistelle, Sittliches Verhalten, 20.7.1936.

66 Dies bekunden staatliche wie kirchliche Akten. Gröber spricht von einer „Nymphomanin“. Aktennotizen Gröber zu G. (hs), o.D. EAF Nb 8/140.

67 So wurde - allerdings erst viele Jahre später - festgestellt: „Sie selbst war bei der ganzen Liebesaffäre [...], wie sie selbst zugibt, nicht etwa der verführte, sondern vielmehr der aktive Teil“. Gerichtsärztliches Gutachten an Staatsanwaltschaft Freiburg (11 S.), 16.7.1936. StAF G $1167 / 1$ Nr. 773 . 
die divergierenden erkenntnisleitenden Interessen der Protokollführenden auch den Fokus der Aussagen diktierten.

Inwieweit die Erfahrungen der Jahre 1924 und 1925 negativ auf die Entwicklung beider Persönlichkeiten eingewirkt haben - bei Ottilie G. kommt es zu rasch wechselnden Partnerschaften, verbunden mit der Unfähigkeit zu festen Bindungen, bei Lorch sieht das kaum anders aus - lässt sich ebenfalls nur vermuten. Unklar ist bereits, ob der Missbrauch des Jahres 1924 Ausgangspunkt oder lediglich Etappe zweier immer weiter eskalierender Sexualbiographien war. Die vom Täter an den Tag gelegte Raffinesse lässt vorgängige Erfahrungen immerhin vermuten; aber auch die Betroffene scheint kein unbeschriebenes Blatt gewesen zu sein. ${ }^{68}$ Zumindest für sie muss aber doch angenommen werden, dass die Erfahrungen - Gewalt, Schweigegebote, die anfängliche Unfähigkeit, sich jemandem (selbst der Stiefmutter gegenüber) zu offenbaren, die (jedoch widerstrebende) Hinnahme der versuchten Abtreibung, die Geburt des ersten Kindes ohne familiären Beistand in fremder Umgebung, Krankheit, Wegnahme und Tod des Kindes - traumatisierende Wirkung besaßen. ${ }^{69}$ Eine Zerrüttung der psychischen Integrität scheint von daher erst die Summierung der vielfältigen negativen Erfahrungen, Beziehungs- und Lebenskrisen herbeigeführt zu haben. Auch beim Täter verweisen die (spröden Personal-)Akten auf erhebliche psychische und körperliche Auswirkungen des Missbrauchs bzw. der kirchlichen Ahndung (Arbeitsbeschränkung, Wegsperrung, engmaschige Aufsicht etc.). ${ }^{70}$

Die Wertung des kirchlichen Umgangs mit dem Fall (und fortwirkend: auch mit den späteren Abweichungen von der kirchlichen Sexualnorm) bleibt ambivalent. Zum einen, weil die Sachlage weder damals klar zutage trat noch heute wirklich geklärt werden kann. Zum anderen wechselten die Akteure und - im zeitlichen Fortgang - auch die zu berücksichtigenden Faktoren. Dem Ordinariat lag weder 1925 noch später jene Schilderung des Hergangs vor, die Ottilie G. 1936 der Gestapo gegenüber zu Protokoll gab (oder gegeben haben soll), sehr wahrscheinlich auch kein sonstiger Hinweis auf eine sexuelle

68 Ein Indiz dafür, dass Ottilie G. von Jugend an als "gefährdet" galt, ist, dass sie ab etwa dem 16. Lebensjahr von der Fürsorge betreut wurde, die nicht nur eine gewisse ,Aufsicht' zu garantieren versuchte, sondern sich auch darum kümmerte, dass Ottilie G. in geregelte Arbeit und also - in den wirtschaftlich schwierigen 192oer Jahren - in Lohn und Brot kam. Im Hintergrund standen möglicherweise Erfahrungen während ihrer Berufsausbildung im sozial-medizinischen Bereich - ob auch bereits (und tatsächlich) Missbrauch? Gröber deutet dies nur dunkel (und in problematischer Wortwahl) an, wenn er mehrfach und pointiert die wohl fremde Zuschreibung „das blonde Gift“ zitiert.

69 Der hin und wieder später von ihr genommene Rekurs auf Lorch scheint dies nahezulegen.

70 Dieser Aspekt könnte anhand der Akte eingehend dargestellt werden. 
Gewaltausübung Lorchs gegen den Willen der Betroffenen (Vergewaltigung; offenkundiger Missbrauch). Für das Ordinariat lag es also durchaus nahe, von Einvernehmlichkeit bzw. „Verführung“ Ottilie G.s durch Lorch (so Gröber) ausgehen. Weitere Zölibatsverstöße Lorchs (mit erwachsenen Frauen) in den folgenden Jahren mussten dieses Bild verfestigen. Gleichwohl war Gröber 1925 klar, dass es sich bei Ottilie G. um eine „Gefährdete“ handelte, so dass er eine besondere Verantwortlichkeit Lorchs erkannte. Diese nahm Gröber später rückblickend - auch für das weitere Schicksal Ottilie G.s (mit) in Anspruch.

Lorch wurde 1925 aus der Pastoral abgezogen, zunächst kirchlich inhaftiert und (auf Zeit) unter besondere Aufsicht gestellt. Die Frage, ob ihm damals wie dann sicher 1931 - auch ein kirchlicher Prozess gemacht wurde, ist wohl zu verneinen. Eine Weiterverwendung im Erzbistum kam jedoch vorerst nicht mehr in Frage. Wenn das Ordinariat als zentralen Grund dafür wiederholt "Diffamation" angab, so zielte dies in zwei Richtungen: Zum einen war mit dem öffentlichen Bekanntwerden des Falles ein gedeihliches seelsorgerliches Wirken Lorchs für die Zukunft ausgeschlossen, zum anderen bestand - zumal in nationalsozialistischer Zeit - die Gefahr der Ausschlachtung des Falles gegen die Kirche insgesamt. ${ }^{71}$ Insofern dominierte im Handeln des Ordinariats das Motiv des Schutzes der Institution Kirche und die Integrität der Seelsorge. ${ }^{72}$ Die Beschneidung der priesterlichen Betätigungsmöglichkeiten Lorchs war aber auch als Strafmaßnahme, als Zeit der Beobachtung und Bewährung und insofern als prophylaktische Vorsorge gedacht. Nicht zuletzt wurde durch die räumliche Entfernung Lorchs der weitere Kontakt mit der Betroffenen (und eine mögliche Fortsetzung des Missbrauchs - in der Perspektive des Ordinariats: der Beziehung) erschwert.

Wenn das Ordinariat sich nach der "Auszeit" Lorchs für die Vermittlung einer Stelle einsetzte, so kam es damit zweifelsohne seiner Fürsorgepflicht als Arbeitgeber nach. Dies war kein leichtes Unterfangen, denn die angefragten Bistümer und Orden wurden über Lorchs „Fehltritt“ informiert. Bei der Stellenwahl scheint eine Rolle gespielt zu haben, ob eine Beaufsichtigung Lorchs

\footnotetext{
71 Auch wenn 1933 noch keine Schauprozesse wegen Sittlichkeitsdelikten von Klerikern geführt wurden, schlachtete die (nationalsozialistische) Presse derartige Fälle entsprechend aus.

72 „Wir haben die Frage ihrer Wiedereinstellung in der Erzdiözese eingehend und wohlwollend geprüft, kamen aber zu unserem Bedauern zur Überzeugung, daß wir Ihnen jetzt, nachdem erst 5 Jahre seit Ihrem großen aufsehenerregenden schweren Fehltritt verflossen sind, eine Anstellung nicht geben können, ohne Gefahr zu laufen, daß die Sache wieder zu Ihrer Diffamation und zum großen Schaden der Seelsorge aufgerührt würde“. Ordinariat Freiburg an Lorch, 5·9.1930.
} 
garantiert werden konnte. Es lag im ureigensten kirchlichen Interesse, Wiederholungen zu verhindern. ${ }^{73}$

Weit weniger ausgeprägt als der Blick des Ordinariats auf den Täter war der Blick auf die Betroffene. Dies schon allein deshalb, weil das Erzbistum gegenüber dem Täter unmittelbare Rechte und Pflichten hatte, und zwar nicht nur im geistlichen Sinne, sondern (als Arbeitgeber) auch im bürgerlichen, während die Betroffene sich dem unmittelbaren Zugriff des Ordinariats de iure und de facto entzog. Gleichwohl verschwand diese nicht vom ,Bildschirm' des Freiburger Ordinariats. Das mag damit zusammenhängen, dass sie schon zuvor im Fokus der kirchlichen Fürsorgearbeit gestanden hatte, dass also ein Sonderfall vorlag. Schon die Frage Gröbers an Ottilie G., ob er die Sache in die Hand nehmen dürfe, ist - zumal für die damalige Zeit - beachtlich. Ob die ,Lösung Entfernung Lorchs, Erzwingung eines radikalen Abbruchs der Beziehungen dem Wollen und Wünschen der Betroffenen entsprach, sei dahingestellt. ${ }^{74}$ Zur Perspektive auf die Betroffene gehörte nicht nur, dass man sich 1925 unmittelbar um das kranke Kind kümmerte. Dazu kamen in den darauffolgenden Jahren punktuell sozial-caritative Unterstützungen, die zeitweise Organisation einer persönlichen Betreuung und - immer wieder - Kriseninterventionen. Zeugnisse hierüber sind freilich nicht in der Personalakte des Täters zu finden.

Das Thema Verantwortlichkeit erweist sich insgesamt als schwierig. Wie wir sahen, beginnt Verantwortlichkeit im weiteren (wenn auch nicht kausalen) Sinn mit dem längeren Auswahl- und Aufnahmeprozess in den kirchlichen Dienst, führt über Qualifizierungs- und Ausbildungsstufen bis hin zu der - faktisch in der Regel jedoch schwierig zu handhabenden - Dienstaufsicht des Pfarrers über die Kapläne, des Dekans über die Pfarrer. In der Praxis war ein schmaler Weg zu suchen zwischen aufmerksamer Beobachtung und denunziatorischer Überwachung des, Untergebenen'. Auf der untersten Ebene erwies sich dies als besonders schwierig. Denn hier agierten alle im durchaus ambivalenten Klima eines zwar hierarchisch geordneten, aber doch - im besten Fall - mitbrüderlich-vertrauensvoll gestalteten Arbeitsbereichs, der gleichzeitig gemeinsamer (quasi-familiärer) Lebensraum war. Dass hier mitunter Hemmungen vorlagen, ist begreiflich. Die Informationsbeschaffungsmöglichkeiten der kirchlichen Behörde (in unserem Fall: des Erzbischöflichen Ordinariats) waren selbst in diesem Rahmen äußerst begrenzt. Dies gilt letztlich auch für die (zudem höchst unzulänglichen) Handlungsoptionen gegenüber dem Täter.

73 Vgl. das obige Verbot, eine Stelle an einer höheren Mädchenschule anzunehmen.

74 Unklar ist, inwieweit man von einer beiderseitigen Einwilligung in das Tatgeschehen sprechen kann oder muss. 